\title{
Multiple gene mutations in patients with type 2 autoimmune pancreatitis and its clinical features
}

\author{
FENG DONG ${ }^{*}$, QING-QUAN CHEN ${ }^{*}, Z E-H A O ~ Z H U A N G^{3}$, QING-LIANG HE \\ FENG-QING WANG ${ }^{5}$ QI-CAI LIU ${ }^{5}, H E-K U N L I U^{6}, Y U W A N G^{2}$ \\ *These authors contributed equally to this manuscript. \\ 'Department of Radiation Oncology, Fujian Medical University, Fuzhou, PR China \\ ${ }^{2}$ Department of Laboratory Medicine, Medical Technology and Engineering College, Fujian Medical University, Fuzhou, PR China \\ ${ }^{3}$ Department of Gastroenteropathy, Fujian Medical University, Fuzhou, PR China \\ ${ }^{4}$ Department of Surgery, Fujian Medical University, Fuzhou, PR China \\ ${ }^{5}$ Department of Laboratory Medicine, the First Affiliated Hospital, Fujian Medical University, Fuzhou, PR China \\ ${ }^{6}$ Department of Cell Biology and Genetics, Fujian Medical University, Fuzhou, PR China
}

\begin{abstract}
Background: It is now clear that there are two histological types (type 1 and type 2) of autoimmune pancreatitis (AIP). The histological substance of type 1 AIP is known as lymphoplasmacytic sclerosing pancreatitis $(L P S P)$ or traditional AIP, and type 2 AIP is characterized by distinct histology called idiopathic duct centric pancreatitis (IDCP). Serum IgG4 increase is considered as a marker for type 1 AIP. Far less is known about type 2 and it lacks predicting markers, so it easily leads to missed diagnosis and misdiagnosis.

The aim of this study was to describe multi-gene mutations in patients with type 2 AIP and its clinical features.

Material and methods: Three unrelated patients with type 2 AIP, 10 cases with type 1 AIP, 15 cases with other chronic pancreatitis and 120 healthy individuals were studied. The mutations and polymorphisms of 6 genes involved in chronic pancreatitis or pancreatic cancer - PRSS1, SPINK1, CFTR, MEN1, PKHD1, and mitochondrial DNA - were sequenced. Information of clinical data was collected by personal interview using a structured questionnaire.

Results: Novel mutations were found in the genes encoding for MEN1 (p.546 Ala > The) and PKHD1 (c. $233586 A>G$ and c. $316713 C>T$ ) from patients with type 2 AIP. What is more, the serum TCR (T cell receptor) level is relatively higher in patients with type 2 AIP than in patients with type 1 $A I P$ and other chronic pancreatitis or normal controls. Weight loss was the major manifestation and no patients had extrapancreatic involvement in type 2 AIP.

Conclusions: Type 2 AIP may occur with multi-gene mutations. For screening purposes, it is more reasonable to evaluate TCR levels in serum.
\end{abstract}

Key words: autoimmune pancreatitis, type 2, gene mutations, serum TCR.

(Centr Eur J Immunol 2014; 39 (1): 77-82)

\section{Introduction}

Most of the early literature pertaining to autoimmune pancreatitis (AIP) came from Japan [1-3]. According to these criteria, AIP is classified into 2 types [4-9]. The histological substance of type 1 AIP is known as lymphoplasmacytic sclerosing pancreatitis (LPSP), and type 2 AIP is characterized by distinct histology called idiopathic duct centric pancreatitis (IDCP). IgG4 positive plasma cells are considered a marker for type 1 AIP, it can be detected in the pancreas and a variety of other tissues and increased serum IgG4 were non-invasive biomarkers [1, 4]. Type 2 AIP is generally seronegative and lacks other organ involvement in contrast to type 1 AIP. Type 2 AIP more easily leads to missed diagnosis and misdiagnosis. Histological differentiation is becoming more important for diagnosing type 2 AIP. However, a surgically resected specimen can only be obtained from a patient who was

Correspondence: Dr. Qi-cai Liu, Department of Laboratory Medicine, First Affiliated Hospital, Fujian Medical University,

20 Cha Zhong Road, Taijiang, Fuzhou 350005, China, tel. 86-591-87981972, e-mail: 1qc673673673@163.com

Liu HK, PhD, Department of Cell Biology and Genetics, Fujian Medical University, Fuzhou 350005, China, tel. 86-591-87982733, e-mail: lqclqc2013@163.com 
misdiagnosed with pancreatic cancer and had a surgical operation $[10,11]$. Further studies are needed to clarify if cases with normal serum IgG4 are a precursor of type 1 or type 2 AIP or other diseases, and its molecular mechanism is still unknown.

Genetic factors have been identified in patients with chronic pancreatitis (CP) and these factors are believed to play an important role in the pathogenesis of CP. Mutations in protease serine 1 (PRSS1) (OMIM 276000), cystic fibrosis transmembrane conductance regulator (CFTR) (OMIM 602421), and pancreatic secretory trypsin inhibitor (SPINK1) (OMIM 167790), were causally linked to the pathogenesis of CP. Although there is no direct evidence of vascular involvement in the pancreas of patients with the A3243G mutation, it is known that the organ is susceptible to ischemic injury, and that perturbations of the systemic and pancreatic microvascularization play a significant role in the pathogenesis of pancreatitis [12, 13]. Moreover, polycystic kidney and hepatic disease 1 (PKHD1) gene mutation may be a genetic factor for pancreatitis. Williams produced a mouse model of autosomal recessive polycystic kidney disease by replacing exons 1-3 of Pkhd1 with a lacZ reporter gene utilizing homologous recombination. Dilatation of pancreatic exocrine ducts was uniformly seen in Pkhd1 (lacZ/lacZ) mice, with pancreatic cysts arising less frequently. The expression of beta-galactosidase, $\mathrm{Pkd} 1$, and $\mathrm{Pkd} 2$ was reduced in the kidneys of Pkhd1 (lacZ/lacZ) mice compared with wild-type littermates. These results indicate that deletion of exons 1-3 leads to loss of Pkhd1 expression and results in kidney cysts, pancreatic cysts. Therefore, there are reasons to believe that there is an association between $P K D H 1$ gene mutation and pancreatitis [14].

\section{Material and methods}

\section{Patients and controls}

A total of three patients with type 2 AIP, ten cases with type 1 AIP, 15 cases with other chronic pancreatitis and 120 healthy individuals in the past three years were included in the study, there was no history of tobacco smoking or alcohol consumption in these patients. All patients originated from the Han ethnicity in the mainland of China. AIP diagnostic criteria: I. Pancreatic imaging studies show diffuse narrowing of the main pancreatic duct with an irregular wall (more than 1/3 of length of the entire pancreas). II. Laboratory data demonstrate abnormally elevated levels of serum gamma globulin and/or IgG, or the presence of autoantibodies. III. Histopathologic examination of the pancreas shows fibrotic changes with lymphocyte and plasma cell infiltrate. For diagnosis, criterion I (pancreatic imaging) must be present with criterion II (laboratory data) and/or III (histopathologic findings).

\section{DNA extraction and molecular genetic analysis}

Genomic DNA was extracted from peripheral blood and other tissue specimens using a QIAamp DNA mini kit (Qiagen, Germany). Six genes involved in pancreatitis/pancreatic cancer - PRSS1, CFTR, SPINK1 and multiple endocrine neoplasia 1 (MEN1), polycystic kidney and hepatic disease 1 (PKHD1) and mtDNA - were sequenced according to references [15].

The PCR methods and primers for PRSS1, CFTR, SPINK1 and MEN1 genes were the following reference [15]. And the primers of $P K D H 1$ gene are as follows: 1F: tgt gaa tca gaa tgg gca gtg, 1R: aac aag ccc tga gga aaa agc, 2F: tgg gga tga tttt atg caa gg, 2R: ggt gtt aag gta ttt gct ttt ggg, 3F: tcc tga tga gtg cag ggt ttt, 3R: gca aag cac agc ataccatga, 4F: tac ccc cag gat ctt agc aca, 4R: ttg ctg tga ttc aat tgc cag, 5F: tga aag gtg act gct ggg aat, 5R: aaa ggc aaa ttg taa atg agc ca, 6F: aca ggc ttg tca agg ttt gga, 6R: tgc ttt ggt ttt acc tct ggg, 7F: ggt ctc cac aga gec aag aga, 7R: tta tgg tcc ctc atg agc tgg, 8F: gag cca tga gtg catc cct ac, 8R: gat gcc aaa acc ttc ctt gaa, 9F: cct cet ttg agg cca tta caa, 9R: gca aga ttg gtt ctc atg agg a, 10F: ttg gag tct ttg ggc tta tga a, 10R: aat gga agg ggt cca cat ttt, 51F: ttc cca ctg ggt tgt ttt cac, 51R: aat ggg ttg aaa gag gag cag a, 57F: att gca aat ggt ttg gag tca, 57R: ctt ctg ctt gag att ttg tta caa t. The experimental conditions used to generate the fragment was as follows: $50 \mu \mathrm{l}$ of reaction mixture contained $200 \mathrm{ng}$ of genomic DNA, $10 \mathrm{mmol} / \mathrm{l}$ Tris $\mathrm{HCl}$ (pH 9.0), $50 \mathrm{mmol} / \mathrm{l} \mathrm{KCl}, 0.1 \%$ Triton, $2 \mathrm{mmol} / 1 \mathrm{MgCl}_{2}$, $0.25 \mathrm{mmol} / \mathrm{l} \mathrm{dNTPs}, 100 \mathrm{ng}$ of upstream primer, $100 \mathrm{ng}$ of downstream primer and 3.0 U Taq-DNA polymerase. Cycling conditions included an initial step at $95^{\circ} \mathrm{C}$ for 5 minutes, then 30 cycles at $95^{\circ} \mathrm{C}$ for $30 \mathrm{~s}, 55^{\circ} \mathrm{C}$ for $30 \mathrm{~s}, 72^{\circ} \mathrm{C}$ for 1 minute with a final elongation step at $72^{\circ} \mathrm{C}$ for 10 minutes. The PCR products were purified for sequencing after electrophoresis on agarose gel. For sequencing, a Perkin Elmer Big Dye Sequencing kit (Perkin-Elmer, Shelton, CT, USA) and an ABI PRISM7700 sequencer (Perkin-Elmer ABI, Foster City, CA, USA) were used.

\section{Pancreatic tissue pathology and electron microscopy}

Pancreatic tissue were stained with hematoxylin-eosin (HE), Modified Gomori trichrome (MGT), NADH-tetrazolium reductase (NADH-TR), Periodic Acid-Schiff stain (PSA) and IgG4 special dye.

\section{Detection of serum TCR}

Detection of serum TCR was done with ELISA kits (R\&D Systems, Minneapolis, MN, USA).

\section{Results}

\section{Clinical data of the patients with type 2 AIP}

The patients were women with an average age of 43.7 years $(38,49$, and 44). Common characteristics: significant 
Table 1. Clinical data of patients with type 2 AIP, type 1 AIP, chronic pancreatitis, and normal controls

\begin{tabular}{|c|c|c|c|c|c|c|}
\hline \multirow{2}{*}{ Item } & \multicolumn{3}{|c|}{ Type 2 AIP } & \multirow{2}{*}{$\begin{array}{c}\text { Type } 1 \text { AIP } \\
(\bar{x} \pm s)\end{array}$} & \multirow{2}{*}{$\begin{array}{c}\text { Chronic } \\
\text { pancreatitis }\end{array}$} & \multirow{2}{*}{$\begin{array}{l}\text { Normal } \\
\text { controls }\end{array}$} \\
\hline & No. 1 & No. 2 & No. 3 & & & \\
\hline age of onset & 38 & 49 & 44 & $62.6 \pm 12.5$ & $40.5 \pm 13.6$ & - \\
\hline sex, $\%$ men & female & female & female & $90 \%$ men & $60 \%$ men & - \\
\hline abdominal pain & occasionally & - & - & $80 \%$ constantly & constantly & - \\
\hline weight loss (kg/12 months) & 5 & 6.2 & 9 & $8.6 \pm 5.2$ & - & - \\
\hline anti-nuclear antibody $(<1.0)$ & 0.26 & 0.33 & 0.85 & $1.86 \pm 0.59$ & $0.68 \pm 0.32$ & $0.36 \pm 0.19$ \\
\hline $\begin{array}{l}\text { immunoglobulin } \mathrm{G} \\
(\mathrm{IgG})(7-17 \mathrm{~g} / \mathrm{l})\end{array}$ & 12.8 & 14.9 & 6.8 & $27.9 \pm 13.6$ & $15.7 \pm 4.8$ & $12.3 \pm 4.3$ \\
\hline IgG4 (0.08-1.40 g/1) & 1.05 & 0.12 & 0.89 & $12.45 \pm 8.10$ & $0.78 \pm 0.30$ & $0.56 \pm 0.28$ \\
\hline Trypsin (2-8) nmol/1 & 19.85 & 8.55 & 7.74 & $4.1 \pm 2.3$ & $24.9 \pm 10.7$ & $6.65 \pm 3.4$ \\
\hline Dispose and lapse & $\begin{array}{l}\text { postoperative } \\
\text { prednisolone } \\
35 \mathrm{mg} / \mathrm{d} \text {, } \\
\text { improvement }\end{array}$ & $\begin{array}{l}\text { prednisolone } \\
40 \mathrm{mg} / \mathrm{d}, \\
\text { improvement }\end{array}$ & $\begin{array}{l}\text { prednisolone } \\
40 \mathrm{mg} / \mathrm{d} \text {, } \\
\text { improvement }\end{array}$ & - & - & - \\
\hline
\end{tabular}

weight loss (5-9 kg/12 months) and without serum IgG4 increase (Table 1).

Serum TCR of type 2 AIP was $896.3 \mathrm{pg} / \mathrm{ml}$ and it did not significantly change during, prior to and post glucocorticoid treatment, but it was significantly higher than in type 1 AIP (521.6 pg/ml), chronic pancreatitis group (603.8 pg/ $\mathrm{ml}$ ) and normal controls (Fig. 1).

It is showed that serum TCR in type 2 AIP is significant higher than in type 1 AIP and chronic pancreatitis group and normal controls.

\section{Molecular genetic analysis}

Heteroplasmy for the A3243G mutation in mtDNA was found in one of the affected patients (Fig. 2A). And novel mutations of MEN1 (p.546 Ala > The) (Fig. 2B) and PKHDI (c. $233586 \mathrm{~A}>\mathrm{G}$ and c. $316713 \mathrm{C}>\mathrm{T}$ ) (Figs. $2 \mathrm{C}, \mathrm{D})$ gene were found in the pancreatic tissue and blood samples. In addition, in the affected type 2 AIP patients, no mutations were found in the genes coding for PRSS1, SPINK1 and CFTR. These mutations were not found in the normal controls and other patients.

\section{Pathological analysis}

Histopathologic examination of the pancreas reveals a large number of inflammatory cell infiltrations (mainly neutrophils) (Figs. 3A) and IgG4 negative plasma cells (Figs. 3B), and exhibits interstitial fibrosis and acinar cell atrophy in later stages. However, localization and the degree of duct wall infiltration are variable.

\section{Discussion}

Recently, two types of AIP have been distinguished [49]. They share the symptomatology and some histopatho-

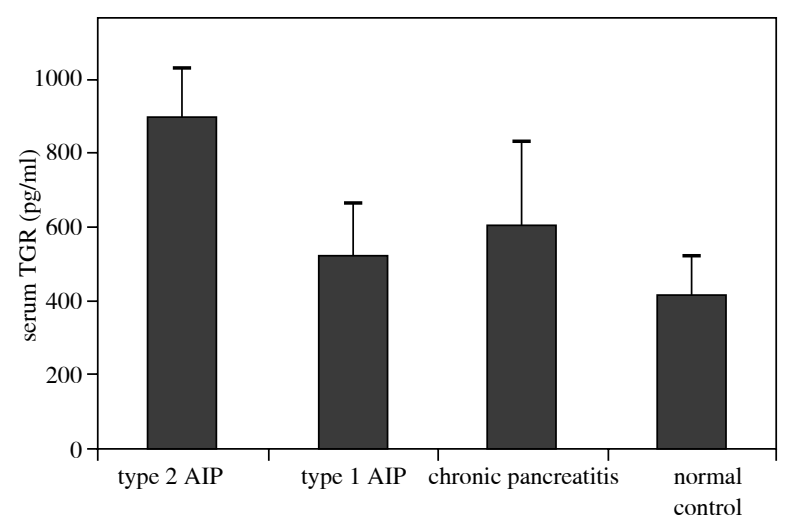

Fig. 1. Different serum TCR in type 2 AIP, type 1 AIP, chronic pancreatitis, and normal controls

logical features such as periductal lymphoplasmacytic infiltrate and storiform fibrosis, but differ in a particular duct change, called granulocytic epithelial lesion, which characterizes type 2 AIP. In addition, type 2 AIP usually has no or very few IgG4-positive plasma cells. Type 2 AIP patients frequently show an association with inflammatory bowel disease and usually lack serological elevation of IgG4 [16, 17]. The main differential diagnosis of AIP is pancreatic ductal adenocarcinoma. In North America, about $2.5 \%$ of patients with a preoperative diagnosis of pancreatic cancer are diagnosed with AIP postoperatively. In many instances, the diagnosis of AIP can be made by imaging together with serological markers. In difficult cases, particularly in type 2 AIP, the diagnosis has to be established by core needle biopsy [11, 18]. Type- 2 AIP tends to have focal features and it is more commonly surgically 
Feng Dong et al.

A

60

70

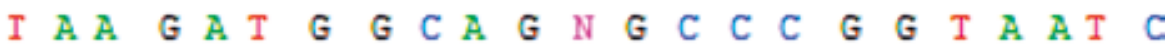

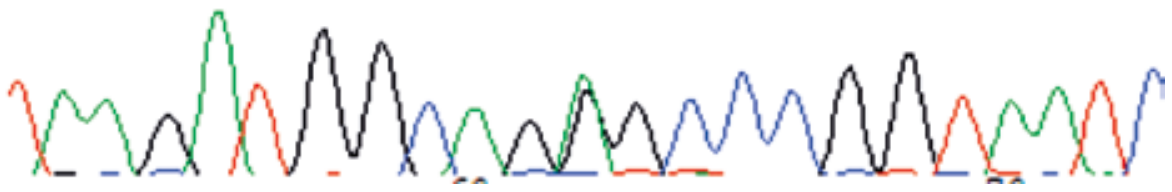

B

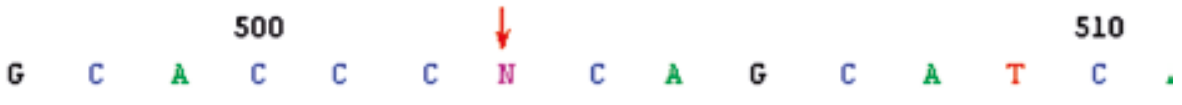

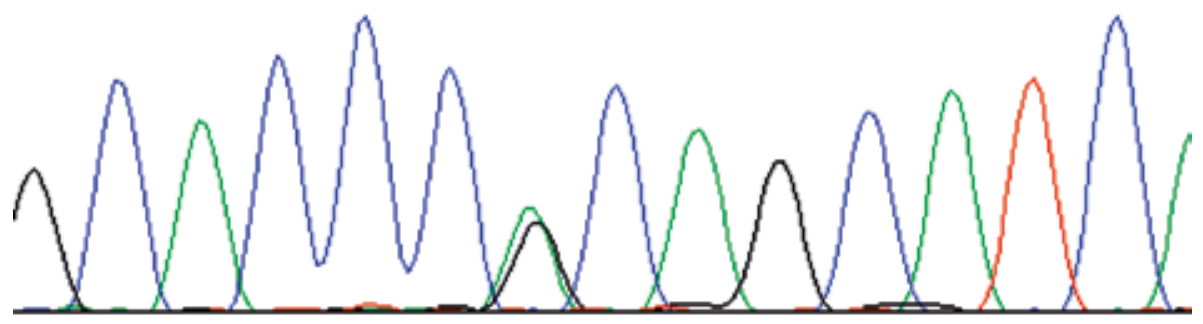

A $\quad G \begin{array}{cccccccccccccccccccccc}500 & G & C & A & C & C & C & G & C & A & G & C & A & T & C & A & C\end{array}$

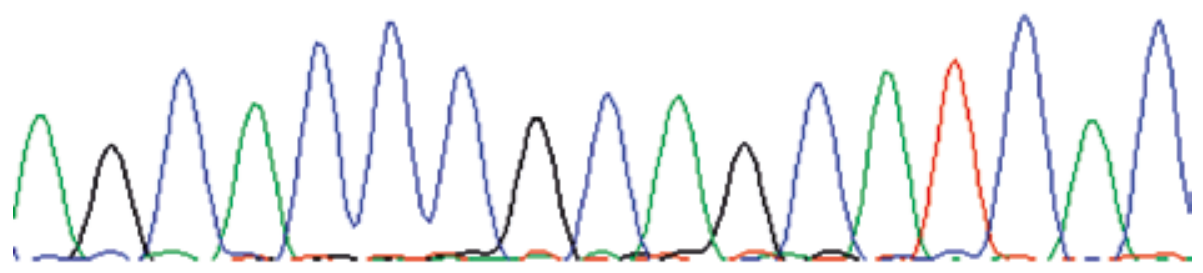

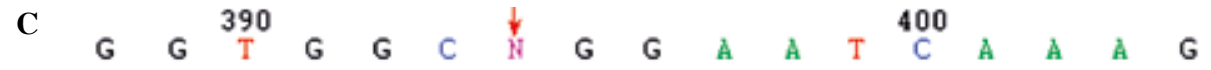

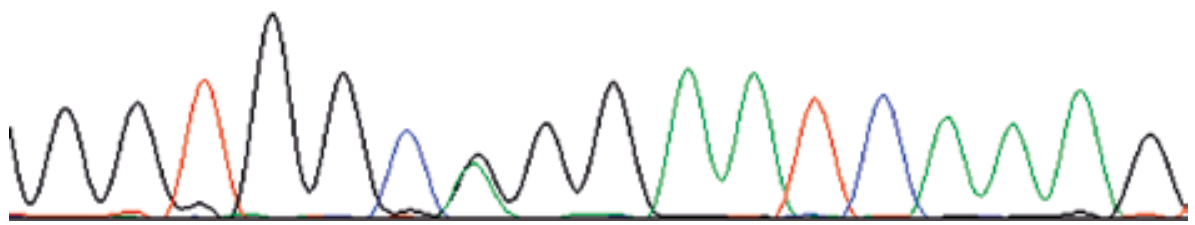

D

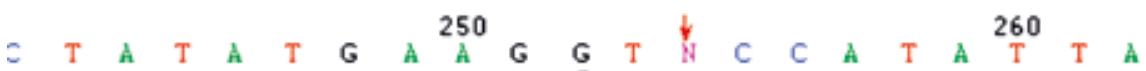

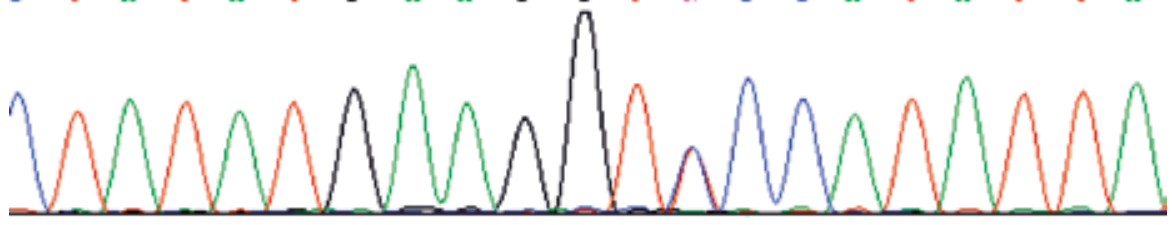

Fig. 2. Sequencing of gene mutations of patients with type 2 AIP. A) sequencing of mitochondrial m.A3243G mutation of blood sample of patients with type 2 AIP (No.1); B) sequencing of MEN1 gene mutation (p.546 Ala > The) of blood samples of patients with type 2 AIP(No.1, No.3 ); C and D) sequencing of PKHD1 gene silent mutation c. 233586 A $>$ G (No. 1 and No. 2) and c. 316713 C>T (No. 2 and No. 3) of blood samples of patients with type 2 AIP 

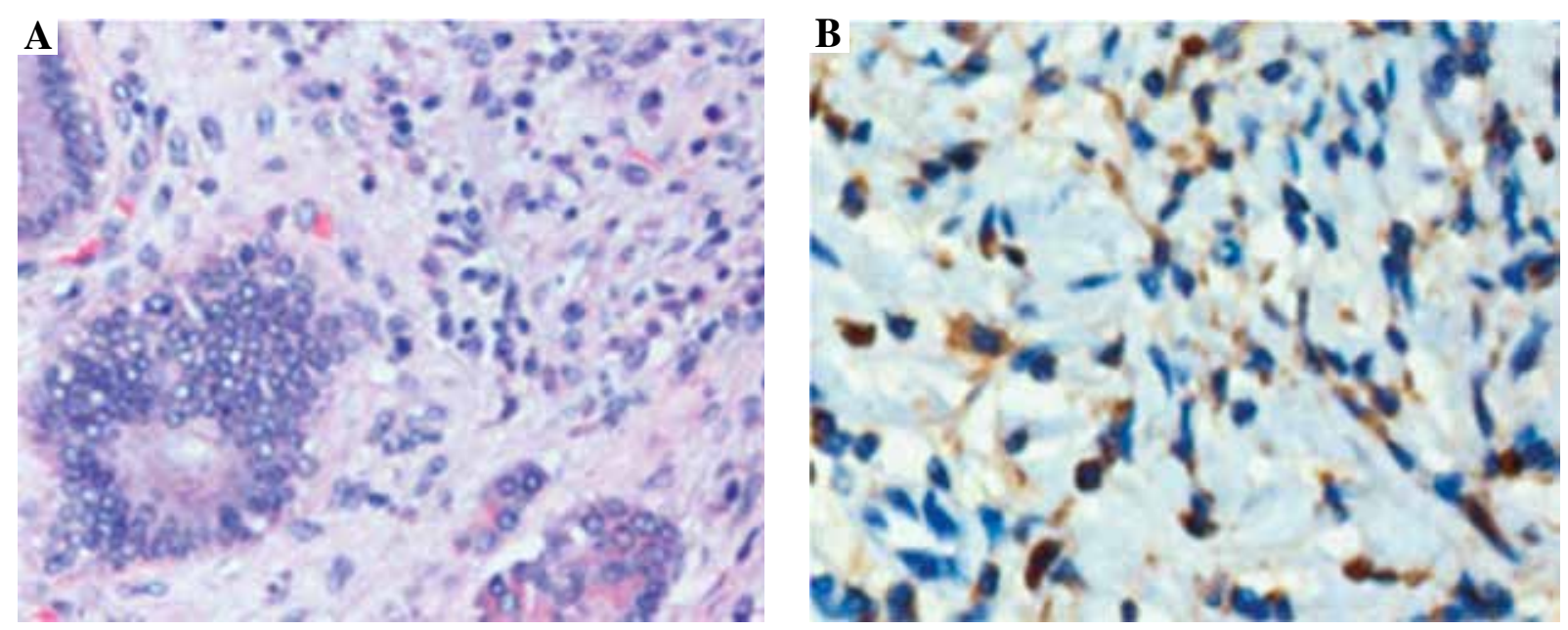

Fig. 3. Histopathologic examination of the pancreas (magnification $400 \times$ ). A) large number of inflammatory cell infiltrations (mainly neutrophils). B) IgG4 staining, positive plasma cells $<10 \%$

resected due to the diagnostic difficulty. Although endocrine and exocrine functions of the pancreas are frequently impaired in type 1 AIP, the functions are unknown.

Genetic analyses have identified a specific gene for hereditary pancreatitis and other types of chronic pancreatitis. The first gene is PRSS1, some PRSS1 mutations enhance trypsinogen autoactivation, and other mutations may render some patients more susceptible to pancreatitis in the presence of other insults to the pancreas. Thus, SPINK1 and CFTR genes have been involved in idiopathic recurrent acute pancreatitis and chronic pancreatitis [19-23]. Several authors have paid attention to particular cases of pancreatitis of an autoimmune pattern and some of the cases were associated with DRBI*0405-DQB1*0401. Parkdo reported that substitution of aspartic acid at the $57^{\text {th }}$ position of haploid DQ $\beta 1$ of the histocompatibility leukocyte antigen is closely related to the recurrence of AIP [15, 24, 25].

In this study, non-hyper-IgG4 was an important clinical feature in patients with type 2 AIP. These findings, however, do raise the possibility that genetic predisposition or histopathology could be useful, particularly when evaluating limited biopsy material. PKHDl gene encodes fibrocystin/polyductin (FPC), a type I membrane protein which is expressed in primary cilia $[26,27]$. The primary cilium is a solitary, non-motile, tubular organelle extending from the apical plasma membrane of the cell [28, 29]. In recent years, it has been proposed that primary cilia sense and transduction multiple stimuli, such as fluid flow, signals initiated by hormones, morphogenes, growth factors and other physiologically active substances present. FPC is localized in primary cilia and acts as a receptor-like protein. This protein is present in fetal and adult kidney cells, and it is also present at low levels in the liver and pancreas. PKHD1 gene c.233586 A $>\mathrm{G}$ and c.316713 C>T mutations may by changing/may change the expression of $\beta$-galactosidase, $\mathrm{Pkd} 1$, and $\mathrm{Pkd} 2$ compared with wildtype littermates and results in pancreatic cysts. The MEN1 gene product, menin, functions as an adaptor protein that is involved in interactions with multiple protein partners. Menin is involved in neuroendocrine cell development and function. Later on, it is active in many cellular processes, including gene transcription regulation, DNA replication, DNA repair, and signal transduction. Clinically, mutations in the MEN1 were associated with better prognosis of pancreatic neuroendocrine tumors [30]. In this study, we also found mutations of PKHD1 and MEN1 gene in type 2 AIP patients. Although the pathogenic mechanism needs further studies, this phenomenon may be used as a secondary diagnosis of AIP.

Our findings suggest that type 2 AIP can present with a variety of clinical phenotypes. In addition, molecular genetic studies have elucidated the molecular mechanisms underlying the pathogenesis of type 2 AIP, which is due to the presence of mutations of MEN1 and PKHD1 gene. Additional clinical studies are required to investigate the clinical heterogeneity and molecular pathogenesis of type 2 AIP.

Authors declare no conflict of interest.

Financially supported by the Natural Science Foundation of Fujian Province (2013J01302), National Natural Science Foundation of China (81201590, 21275028, 81201362), Fujian Medical Innovations (2012-CXB-21), Foundation of Fujian Education Department (JA12143), Outstanding Youth Foundation of Fujian Provincial Higher Education and (JA12133), the Scientific Research Major Program of Fujian Medical University (09ZD013), National High Technology Investigation Project Founda- 
tion of China (2012AA022604) and Fujian province undergraduate innovative experiment project $(265,315,317)$.

\section{References}

1. Yoshida K, Toki F, Takeuchi T, et al. (1995): Chronic pancreatitis caused by an autoimmune abnormality. Proposal of the concept of autoimmune pancreatitis. Dig Dis Sci 40:15611568 .

2. Uchida K, Okazaki K, Konishi Y, et al. (2000): Clinical analysis of autoimmunerelated pancreatitis. Am J Gastroenterol 95: 2788-2794.

3. Kamisawa T, Funata N, Hayashi Y, et al. (2003): Close relationship between autoimmune pancreatitis and multifocal fibrosclerosis. Gut 52: 683-687.

4. Kamisawa T, Chari ST, Giday SA, et al. (2011): Clinical profile of autoimmune pancreatitis and its histological subtypes: an international multicenter survey. Pancreas 40: 352-358.

5. Kamisawa T, Notohara K, Shimosegawa T (2010): Two clinicopathologic subtypes of autoimmune pancreatitis: LPSP and IDCP. Gastroenterology 139: 22-25.

6. Kamisawa T, Okazaki K, Kawa S, et al. (2010): Research Committee for Intractable Pancreatic Disease. Japan Pancreas Society. Japanese consensus guidelines for management of autoimmune pancreatitis: III. Treatment and prognosis of AIP J Gastroenterol 45: 471-477.

7. Kamisawa T, Takuma K, Egawa N, et al. (2010): Autoimmune pancreatitis and IgG4-related sclerosing disease. Nat Rev Gastroenterol Hepatol 7: 401-409.

8. Zamboni G, Luttges J, CAIPelli P, et al. (2004): Histopathological features of diagnostic and clinical relevance in autoimmune pancreatitis: a study on 53 resection specimens and 9 biopsy specimens. Virch Arch 445: 552-563.

9. Sah R P, Chari ST, Pannala R, et al. (2010): Differences in clinical profile and relapse rate of type 1 versus type 2 autoimmune pancreatitis. Gastroenterology 139: 140-148.

10. Ghazale A, Chari ST, Zhang L, et al. (2008): Immunoglobulin G4-associated cholangitis: clinical profile and response to therapy. Gastroenterology 134: 706-715.

11. Kamisawa T, Egawa N, Nakajima H, et al. (2003): Clinical difficulties in the differentiation of autoimmune pancreatitis and pancreatic carcinoma. Am J Gastroenterol 98: 26942699.

12. Gao F, Li YM, Wang CD, et al. (2013): Identification of a novel frame-shift mutation in PRSS1 gene in Han patients with autoimmune pancreatitis. Curr Mol Med 13: in press.

13. Verny C, Amati-Bonneau P, Letournel F, et al. (2008): Mitochondrial DNA A3243G mutation involved in familial diabetes, chronic intestinal pseudo-obstruction and recurrent pancreatitis. Diabetes Metab 34: 620-626.

14. Williams SS, Cobo-Stark P, James LR, et al. (2008): Kidney cysts, pancreatic cysts, and biliary disease in a mouse model of autosomal recessive polycystic kidney disease. Pediatr Nephrol 23: 733-741.

15. Gao F, Li YM, Hong GL, et al. (2013): PRSS1_p.Leu81Met mutation results in autoimmune pan-creatitis. World J Gastroenterol 2013; 19: 3332-3338.

16. Zen Y, Bogdanos DP, Kawa S (2011): Type 1 autoimmune pancreatitis. Orphanet J Rare Dis 6: 82.

17. Detlefsen S, Zamboni G, Frulloni L, et al. (2012): Clinical features and relapse rates after surgery in type 1 autoimmune pancreatitis differ from type 2: a study of 114 surgically treated European patients. Pancreatology 12: 276-283.
18. Detlefsen S, Löhr JM, Drewes AM, et al. (2011): Current concepts in the diagnosis and treatment of type 1 and type 2 autoimmune pancreatitis. Recent Pat Inflamm Allergy Drug Discov 5: 136-149.

19. Takuma K, Kamisawa T, Tabata T, et al. (2011): Short-term and long-term outcomes of autoimmune pancreatitis. Eur J Gastroenterol Hepatol 23: 146-152.

20. Liu QC, Zhuang ZH, Zeng K, et al. (2009): Prevalence of Pancreatic Diabetes in the Patients Carries Mutations or Polymorphisms of PRSS1 Gene in Han Population. Diabetes Technology Therapeutic 11: 799-805.

21. Liu QC, Lin XH, Liu JF, et al. (2012): The -409C/T Genotype of PRSS1 Protects Against Pancreatic Cancer in the Han Chinese Population. Digest Dis Sci 57: 573-579.

22. Chen JM, Férec C (2012): Genetics and pathogenesis of chronic pancreatitis: the 2012 update. Clin Res Hepatol Gastroenterol 36: 334-340.

23. Whitcomb DC, LaRusch J, Krasinskas AM, et al. (2012): Common genetic variants in the CLDN2 and PRSS1-PRSS2 loci alter risk for alcohol-related and sporadic pancreatitis. Nat Genet 44: 1349-1354.

24. Kawa S, Ota M, Yoshizawa K, et al. (2002): HLA DRB10405-DQB10401 haplotype is associated with autoimmune pancreatitis in the Japanese population. Gastroenterology 122: 1264-1269.

25. Freitag TL, Cham C, Sung HH, et al. (2010): Human risk allele HLA-DRB $1 * 0405$ predisposes class II transgenic Ab0 NOD mice to autoimmune pancreatitis. Gastroenterology 139: 281-291.

26. Ward CJ, Hogan MC, Rossetti S, et al. (2002): The gene mutated in autosomal recessive polycystic kidney disease encodes a large, receptor-like protein. Nat Genet 30: 259-269.

27. Onuchic LF, Furu L, Nagasawa Y, et al. (2002): PKHD1, the polycystic kidney and hepatic disease 1 gene, encodes a novel large protein containing multiple immunoglobulin-like plexin-transcription-factor domains and parallel beta-helix 1 repeats. Am J Hum Genet 70: 1305-1317.

28. Praetorius HA, Spring KR. (2005): A physiological view of the primary cilium.Annu Rev Physiol 2005; 67: 515-529.

29. Davenport JR, Yoder BK (2005): An incredible decade for the primary cilium: a look at a once-forgotten organelle. Am J Physiol Renal Physiol 289: 1159-1169.

30. Jiao Y, Shi C, Edil BH, et al. (2011): DAXX/ATRX, MEN1, and $\mathrm{mTOR}$ pathway genes are frequently altered in pancreatic neuroendocrine tumors. Science 331: 1199-1203. 